\title{
EL IMPACTO DE LAS EXPORTACIONES NO PETROLERAS SOBRE EL EMPLEO EN MÉXICO, 1995-2016
}

\author{
Alejandro Molina Vargas* \\ Jorge López Martínez**
}

\section{Resumen}

El objetivo de este trabajo es estimar el impacto de las exportaciones mexicanas no petroleras sobre la evolución del empleo remunerado para el periodo 1995-2016. Para ello, se emplea un modelo de regresión lineal múltiple con información obtenida del INEGI. Los resultados muestran que las exportaciones agroindustriales son las que presentan un mayor dinamismo en la generación de número de empleos remunerados, por encima del resto de la manufactura. Además, el trabajo sugiere la necesidad de dar mayor valor agregado a los productos agropecuarios y a los provenientes de actividades extractivas, puesto que generan un mayor impacto en el empleo, mayor valor en las exportaciones y un encadenamiento con el mercado interno, vía el incentivo de la demanda sobre las inversiones.

\section{Abstract}

The objective of this paper is to estimate the impact of Mexican non-oil exports on the evolution of paid employment for the period 1995-2016. For this, a multiple linear regression model with

* Candidato a Doctor en Ciencias Económicas por la Universidad Autónoma Metropolitana. Correo: alejandromolin@hotmail.com.

** Dr. en Ciencias Económicas por la UAM y profesor de economía en la Licenciatura y Posgrado de la FES Acatlán, UNAM. Correo: j_lopez@comunidad.unam.mx.

Los autores agradecen los comentarios de dos dictaminadores anónimos de Tiempo Económico para mejorar este manuscrito. Cualquier error u omisión remanente es responsabilidad exclusiva de los autores. 
information obtained from INEGI is used. The results show that agroindustrial exports are those that present a greater dynamism in the generation of number of paid jobs, above the rest of manufacturing. In addition, the work suggests the need to give greater added value to agricultural products and those from extractive activities, since they generate a greater impact on employment, greater value in exports and a link with the domestic market, via the incentive of the demand on investments.

Palabras clave: Exportaciones, empleo, política industrial, encadenamientos productivos

Keywords: Exports, employment, industrial policy, productive chains

Clasificación JEL: F16, J23, Q25

\section{Introducción}

Desde el primer tercio de la década de los ochenta del siglo Xx, México cambió de modelo económico, abandonó el modelo de desarrollo hacia adentro y adoptó un modelo de crecimiento enfocado hacia el exterior. El modelo toma impulso cuando México ingresa al Acuerdo General de Aranceles y Comercio (GATT) en 1986 y se consolida con la entrada del Tratado de Libre Comercio de América del Norte (TLCAN) en 1994. El objetivo en la adopción del nuevo modelo, fue que el sector externo fungiera como eje principal para detonar el crecimiento económico. La lógica del cambio consistió en una mayor integración a la economía mundial, donde la competencia internacional obligara a las empresas a ser competitivas en un sistema de mercado libre, y el mecanismo de precios de mercado favoreciera una asignación más eficiente de recursos y un patrón de especialización en el comercio internacional basado en las ventajas comparativas (mano de obra). En resumen, el modelo industrial exportador fungiría como motor del crecimiento económico sostenido en un marco macroeconómico de baja inflación (Villareal y Ramos, 2001; Guillén, 2013). Así, el modelo exportador mexicano tiene como marco de referencia los conceptos de la ventaja comparativa y el modelo de comercio internacional de Heckscher-Ohlin, los cuales señalan que los países se especializan en la producción de los bienes en los que cuentan con una ventaja comparativa. Sin embargo, las contribuciones recientes han subrayado la necesidad de diversificar productos y mercados, con una política industrial moderna que preste más atención a los vínculos externos e internos, que promueva encadenamientos productivos, para fomentar el desarrollo de la economía local.

Los datos muestran que desde los inicios del TLCAN, ha habido una expansión del comercio internacional, en la que destacan el aumento de las exportaciones manufactureras y el aumento de las importaciones de los bienes intermedios, y en menor medida la diversificación de las exportaciones, siendo el mercado estadounidense el principal destino de las exportaciones mexicanas (Gómez, 2012). También se observa el aumento de la inversión extranjera directa (IED), que se ha orientado hacia actividades con alto potencial de crecimiento y ventajas comparativas, como son las plantas maquiladoras (Loría y Brito, 2005) y se dirige a los 
estados que representan mercados potenciales más grandes, como lo son los estados de la frontera norte que facilitan el acceso al mercado de los Estados Unidos y la región del Valle de México, que es la región más poblada de Latinoamérica (Juárez y Ángeles, 2011). Esta apertura ha permitido que la economía mexicana esté cada vez más integrada al sistema de producción internacional. En un estudio reciente se destaca que los ciclos económicos individuales de los tres países del TLCAN están sincronizados con las fluctuaciones industriales de Estados Unidos, siendo éste el que encabeza los cambios en las fluctuaciones del área (Calderón, García y Cruz, 2017).

Desde otra perspectiva, a pesar del auge del comercio internacional, la economía mexicana ha experimentado un lento crecimiento con escasa generación de empleos. Según Carbajal y Almonte (2017), en las últimas tres décadas, a nivel nacional la manufactura ha disminuido sus tasas de crecimiento, lo que indudablemente ha tenido efectos negativos en la generación de empleo formal. Se destaca que esta situación resulta muy preocupante, dado que el sector manufacturero es considerado motor de crecimiento de la economía. Delgado y Márquez (2007) señalan que, con el TLCAN, se ha acentuado la precariedad laboral y que la transnacionalización del mercado laboral ha sido crucial para el mantenimiento y ampliación de la brecha salarial entre México y Estados Unidos. Sobre los resultados del modelo exportador, la literatura es amplia. Rangel (2015) sostiene que, si bien la expansión del comercio exterior de México arroja datos que pueden ser interpretados como un éxito en el periodo del TLCAN, mayor capacidad de exportación así como uno de los receptores de IED más importantes en el mundo, sin embargo, la baja tasa de crecimiento, la desigualdad regional y salarios bajos, no son para nada alentadores.

A diferencia de otros estudios, el objetivo del presente trabajo es estimar el impacto de las exportaciones no petroleras sobre la evolución del empleo para el periodo 1995-2016, mediante un modelo econométrico, utilizando la información que proporciona el Instituto Nacional de Estadística y Geografía (INEGI). El tema es importante, dado que se trata de analizar la dinámica de las exportaciones manufactureras y extractivas en cuanto a la generación de empleos. Para ello, separamos las exportaciones manufacturas en agroindustriales y manufactureras con maquila y sin agroindustria

El trabajo está estructurado de la siguiente manera, después de la introducción, en la parte uno, se hace una revisión de la literatura, en la parte dos, se analizan algunos hechos estilizados, en la parte tres, se corre el modelo econométrico y sus resultados, y finalmente se presentan las conclusiones.

\section{Revisión de la literatura}

Los beneficios del comercio internacional han sido abordados ampliamente en la literatura. En este apartado sólo hacemos mención sobre las exportaciones y el empleo. Gómez (2013), 
resume la importancia del crecimiento de las exportaciones. En el plano macroeconómico, las exportaciones generan divisas, contribuyen a la generación de empleos y al crecimiento del producto interno bruto (PIB). A nivel microeconómico se destaca que, las empresas exportadoras son más eficientes que las que venden principalmente en los mercados domésticos; las empresas exportadoras sirven como conducto para la transferencia de tecnología y generan derramas tecnológicas con encadenamientos hacia atrás y hacia adelante en la economía doméstica; las exportaciones de manufacturas son particularmente altamente intensivas en empleo, especialmente cuando los insumos (trabajo, capital y materias primas,) son surtidos localmente.

La literatura empírica se ha centrado sobre los resultados del TLCAN, por ser el mercado más grande del mundo. Cabe destacar que la oferta de exportación mexicana tiende a ser mixta, es decir, la expansión y la diversificación de las exportaciones. Si sólo se considera al mercado estadounidense, se han diversificado las exportaciones mexicanas (Gómez, 2013), además, existe evidencia de que México está recuperando participación en dicho mercado, desventaja que presenta ante China en los últimos años (Gómez y González, 2017). En este sentido, México aún tiene el pendiente de diversificar mercados, a pesar de que es el país que más tratados y acuerdos comerciales tiene en el mundo. Además, la diversificación de la actividad económica contribuye a aumentar el empleo (Carbajal y Almonte, 2017). Aunque en los primeros años del TLCAN, el segmento más dinámico de las exportaciones ha estado constituido por las manufacturas, especialmente por las del sector maquilador (Fujii y Cervantes, 2010). Según Gómez (2013), una canasta de bienes de exportación más diversificada y la diversificación de los mercados permite a un país enfrentar los retos del desempleo y conseguir los objetivos de estabilidad y crecimiento económico, aunque el concepto de diversificación de las exportaciones puede parecer contradictorio con el de ventaja comparativa, la especialización en pocos productos de exportación expone a un país a una mayor inestabilidad en los ingresos de exportación, los cuales pueden empeorar cuando los productos implicados están sujetos a un deterioro secular de sus términos de intercambio.

Desde otra perspectiva, Bustamante y Sánchez (2017), caracterizan a la economía mexicana, quienes sostienen que si bien, la IED ha dinamizado la industria manufacturera, no se han creado encadenamientos productivos; por un lado, están las grandes empresas dinámicas integradas al exterior y, por otro lado, están la mayoría de pequeñas y medianas empresas desarticuladas, poco dinámicas y que dependen del mercado interno. Además, la estructura de las exportaciones e importaciones se caracteriza en mayor parte por exportar productos manufactureros y por su alta dependencia de insumos importados y de capital, lo que arrastra débilmente al conjunto de la economía (Fujii y Cervantes, 2010; Cisneros y Guerrero, 2014; Cermeño y Rivera, 2016). Sin embargo, Ríos, Contreras y Bustos (2017), señalan que, si bien las exportaciones manufactureras nacionales contienen un porcentaje importante de insumos importados, el valor agregado total se explica por la productividad de fabricación 
global, es decir, que la productividad local no puede generar un valor agregado significativo en México. Por otro lado, las exportaciones mexicanas dependen en lo fundamental y de manera procíclica al ciclo económico de Estados Unidos (Baltazar y Ramos, 2014). Por todo lo anterior, el motor del crecimiento, el sector exportador, tiende a estar desligado del resto de la economía (Vázquez y Avendaño, 2012), y anclado a la dinámica de la economía estadounidense. Por lo que es necesario fortalecer el mercado interno.

En cuanto a la creación de empleos, la discusión es amplia, desde mediados de la década de los ochenta, la generación de empleo a nivel nacional venía perdiendo dinamismo. En el periodo $1985-1990$ tuvo un crecimiento de $0.52 \%$ y de $1.0 \%$ en el periodo $1990-1994$; en particular, las tasas de crecimiento del empleo manufacturero para estos mismos periodos han sido de $0.48 \%,-2.12 \%$ respectivamente (Lechuga y Varela, 2001). La baja tasa de crecimiento del producto (de 1982 a 2010 creció un $2.1 \%$ promedio anual, mientras que el manufacturero de 1982 a 2009 fue 1.88\%), es insuficiente para generar empleo formal; como resultado, el mercado laboral se encuentra deteriorado, de ahí la creciente precarización del empleo (Calderón y Sánchez, 2012). Esta situación se ha transferido a las regiones del país, de acuerdo con Vargas (2003), en el norte del país, el menor dinamismo de la industria maquiladora en la creación del empleo se debe a la mayor presencia de un tipo diferente de establecimientos maquiladores, particularmente las maquiladoras de segunda generación (tecnología que tiende a la automatización), con presencia en las ramas electrónica y de autopartes. Carbajal y Almonte (2017), analizan por gran división de la manufactura el desempeño de la producción y sus efectos en la generación de empleo formal para la región centro de México, quienes identifican que las divisiones de actividad que integran las ramas más tecnificadas, con mayores procesos de innovación y altamente exportadoras, presentan una menor elasticidad ingreso del empleo, lo que explica la escasa generación de empleo.

Si se analiza por tipo de empleo, en el sector manufacturero, se observa un aumento del empleo no calificado que el del empleo calificado (Fujii y Cervantes, 2010; Calderón, Ochoa y Huesca, 2017), en dicho sector predominan las estructuras oligopólicas y empresas transnacionales que se instalan en las diversas regiones del país en busca de trabajo no calificado y bajos salarios, lo que a su vez presiona hacia la baja los salarios del trabajo calificado (Calderón, Ochoa y Huesca, 2017). El efecto positivo en el empleo derivado de la liberalización comercial sólo se constata en el aumento en la intensidad laboral de algunas ramas (Fujii y Cervantes, 2010).

\section{Algunos datos sobre exportaciones y empleos en México}

La apuesta de las políticas gubernamentales en materia económica, se centró en el sector externo. En teoría, el comercio internacional permite la especialización en la producción de ciertos productos y obtener mayor eficiencia con la producción a gran escala. Además, el 
comercio aporta beneficios al permitir al país exportar bienes cuya producción se realiza con una relativa intensidad de recursos que son abundantes mientras que importa aquellos bienes, cuya producción requiere una intensidad en la utilización de recursos que son relativamente escasos. Este modelo sería el motor del crecimiento económico y causa en la generación de empleos bien remunerados, así como de bienes de importación de mejor calidad a precios competitivos, debido a la mayor competencia internacional, entre otras cosas. Sin embargo, como podremos constatar mediante las gráficas siguientes y el modelo de regresión planteado, el efecto de la apertura comercial sobre la generación de empleos no ha sido el esperado.

Según la clasificación de INEGI, las exportaciones totales se dividen en exportaciones petroleras y exportaciones no petroleras. Dado que las primeras corresponden en mayor medida a actividades derivadas de las exportaciones del petróleo crudo, derivados del petróleo y petroquímica, para el modelo, nos centramos en las exportaciones no petroleras. Las exportaciones no petroleras contienen las exportaciones agropecuarias, manufactureras y extractivas. Dentro las agropecuarias tenemos, ganado vacuno, pimiento, jitomate, frutas y frutos comestibles, aguacates, otras legumbres y hortalizas frescas, entre otras actividades. En las manufactureras se consideran las exportaciones de productos elaborados, tales como alimentos, bebidas y tabacos, textiles, artículos de vestir e industria del cuero, productos metálicos, maquinaria y equipo, en las cual están los productos automotrices, entre otras. Finalmente, las extractivas consideran los minerales metálicos y no metálicos.

La Gráfica 1 muestra la evolución de la contribución de las exportaciones petroleras y exportaciones manufactureras en el PIB. La principal actividad de exportación que contribuye al PIB es la manufactura, su contribución al Producto ha tenido una evolución positiva. Al inicio del periodo aportaba el 19.13\%, mientras que para 2016 su contribución fue de $32.1 \%$. Una de las principales actividades manufactureras de exportación sin duda ha sido la automotriz, la cual ha incrementado su presencia en el país en la última década. En cuanto a la contribución de las exportaciones petroleras al producto, las cuales presentaron algunos vaivenes, pero que por lo menos de 2001 a 2008 tuvieron un crecimiento importante. A partir de 2011, las exportaciones petroleras han venido disminuyendo, resultado de dos factores: el agotamiento de los yacimientos y la caída en el precio internacional del petróleo. Mientras que en el Cuadro 2 se muestra las exportaciones agropecuarias, agroindustriales y extractivas como porcentaje del PIB. Cabe mencionar que las exportaciones manufactureras contienen las agroindustriales (Alimentos, bebidas y tabaco, Productos Químicos, Productos Textiles y del Cuero). La separación que se hace aquí se debe al planteamiento del modelo ensayado.

La estructura porcentual de las exportaciones se presenta en el Cuadro 1. Son las exportaciones manufactureras con maquila (sin agroindustria) las que mayor participación tienen. Las que han aumentado ligeramente su participación en los últimos años son las agroindustriales y las extractivas. Para 2016 las exportaciones agropecuarias representaron $3.92 \%$, las manufactureras con maquila (sin agroindustria) $86.05 \%$, las agroindustriales $3.82 \%$ y 


\section{Gráfica 1}

Exportaciones petroleras y manufactureras

como \% del PIB, 1995-2016

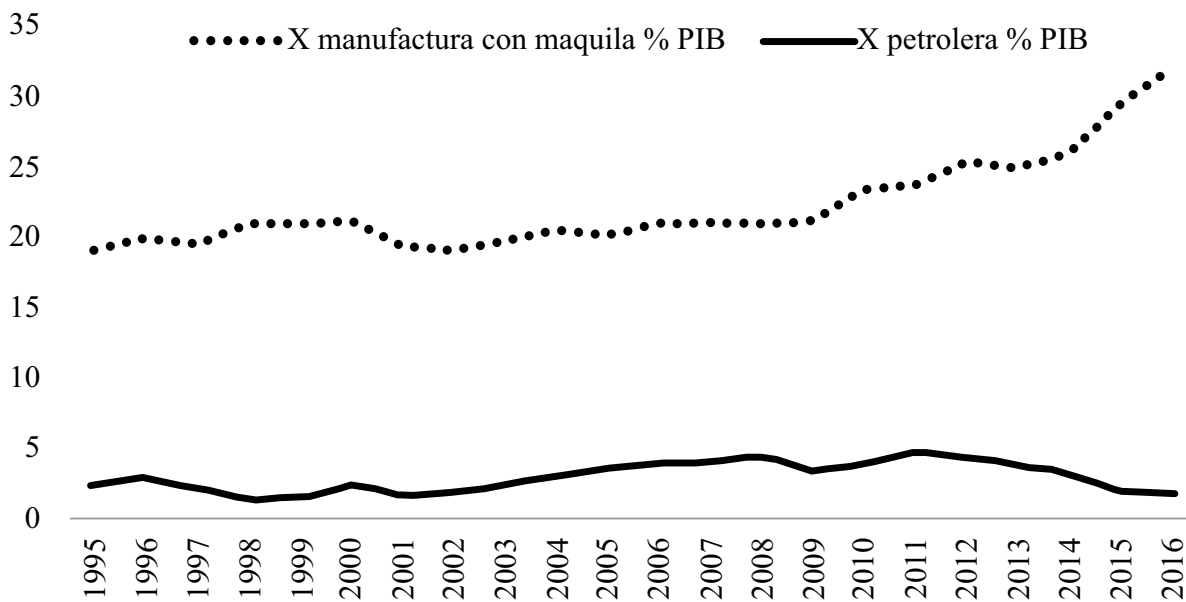

Fuente: Elaboración propia con base en datos de INEGI y Banco Mundial.

\section{Gráfica 2}

Exportaciones agropecuarias, agroindustriales y extractivas como \% del PIB, 1995-2016

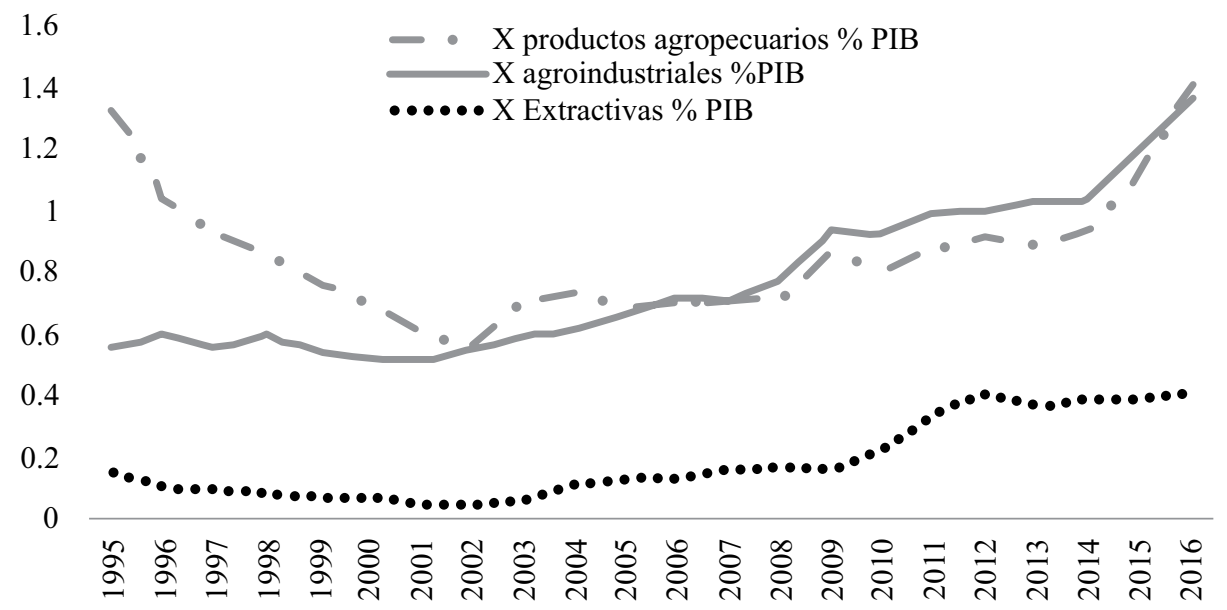

Fuente: Elaboración propia con base en datos de INEGI y Banco Mundial. 
las extractivas $1.17 \%$. Las exportaciones petroleras, las cuales a partir de 2011 han caído de manera importante, pasando de $16.15 \%$ a $5.03 \%$ en 2016.

Cuadro 1. Porcentaje de participación de principales exportaciones con respecto a exportaciones totales, 1995-2016

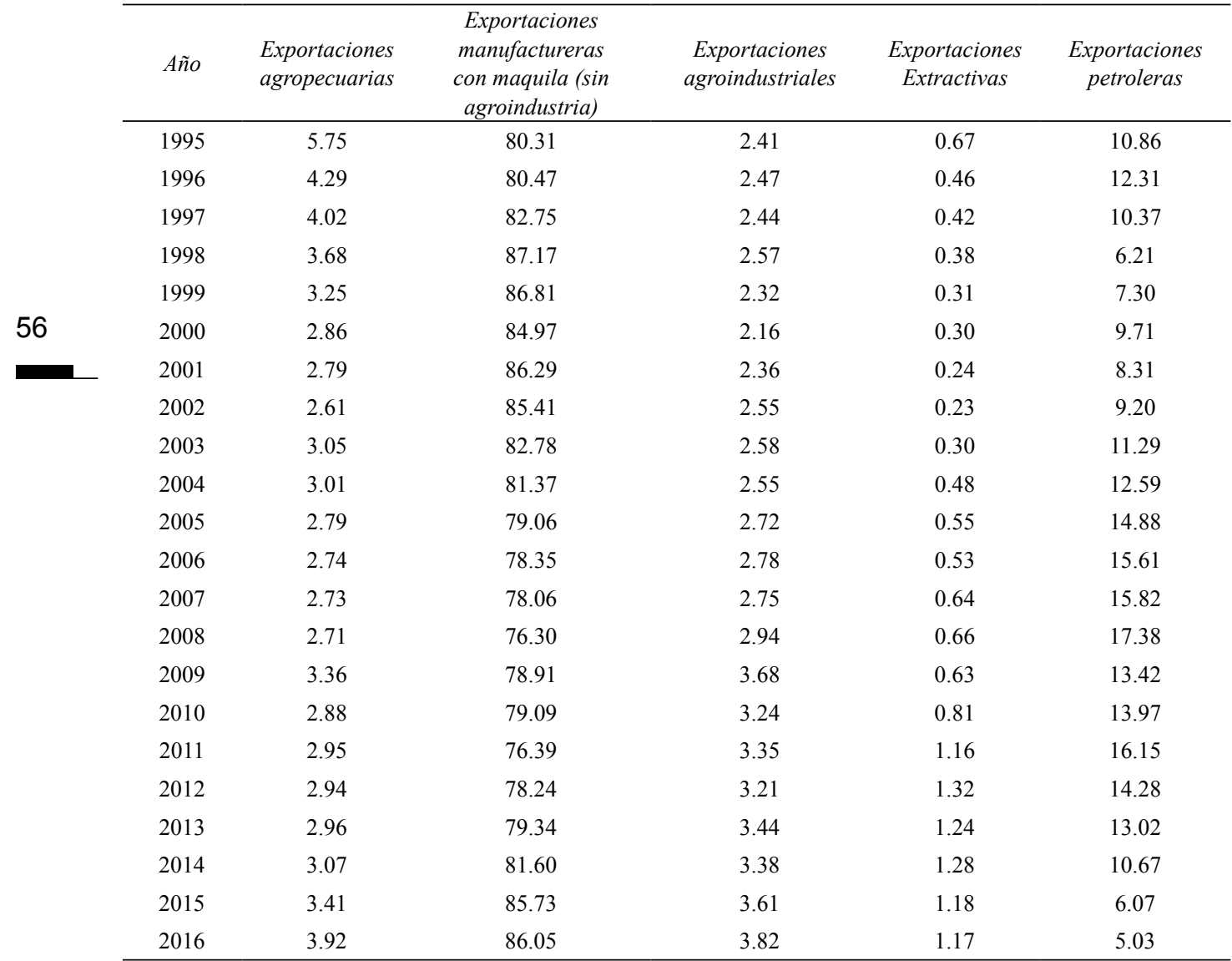

Fuente: Elaboración propia con base en datos de INEGI.

La importancia relativa de cada categoría de las exportaciones se mantiene. Siguen siendo las exportaciones manufactureras las que conforman la mayor parte de las exportaciones totales. En este panorama, se esperaría que fuera precisamente la industria manufacturera de exportación la que tuviera un mayor impacto en la generación de empleos, el modelo de regresión que se realiza en la siguiente sección arrojará luz a este respecto.

En cuanto al Valor Agregado de Exportación de la Manufactura Global (VAEMG) representó en 2016 el 45.9\% de la producción manufacturera global. Es decir, el valor del contenido nacional que se añade a los productos manufactureros que se exportan. La participación de la manufactura mexicana ha aumentado en los últimos años de manera paulatina en el contexto internacional de las cadenas globales de valor (Gráfica 3). 


\section{Gráfica 3}

Participación del Contenido Nacional de las Exportaciones Globales en la Producción Manufacturera Global

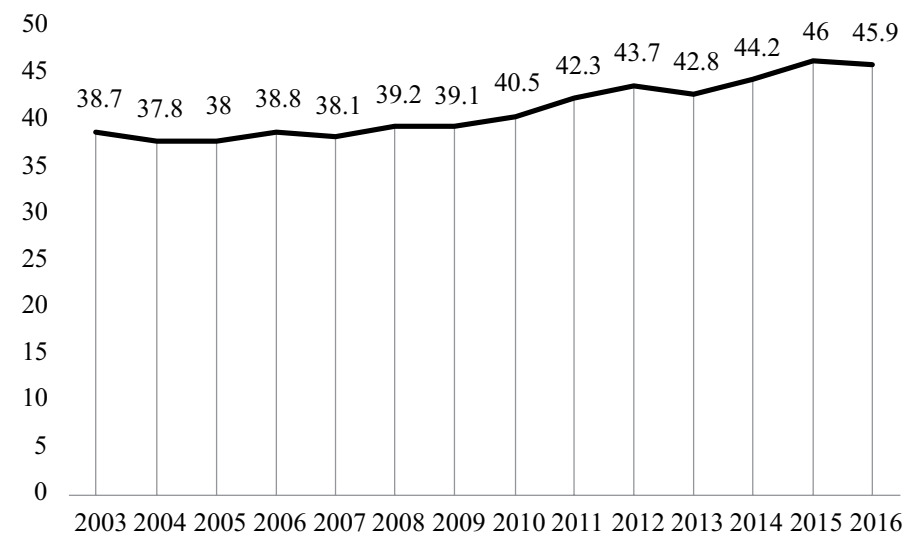

Fuente: Elaboración propia con datos de INEGI.

Finalmente, la dinámica de crecimiento de las principales exportaciones analizadas se presentan en la Gráfica 4. Las exportaciones extractivas son las que han presentado mayor varianza, creciendo hasta el $80 \%$ en 2003 y cayendo en poco más del $25 \%$ en 2008, en promedio, su tasa de crecimiento es de $13.87 \%$. Sin duda el factor principal de esta dinámica se encuentra en las fluctuaciones de la demanda en el mercado internacional. En promedio, las exportaciones agroindustriales crecieron a un ritmo del $10.27 \%$ anual, mientras que las manufactureras sin la agroindustria lo hicieron en $8.5 \%$, aunque es importante señalar que desde 2015 se han estancado.

\section{Gráfica 4}

Dinámica de crecimiento de las principales exportaciones, 1996-2016

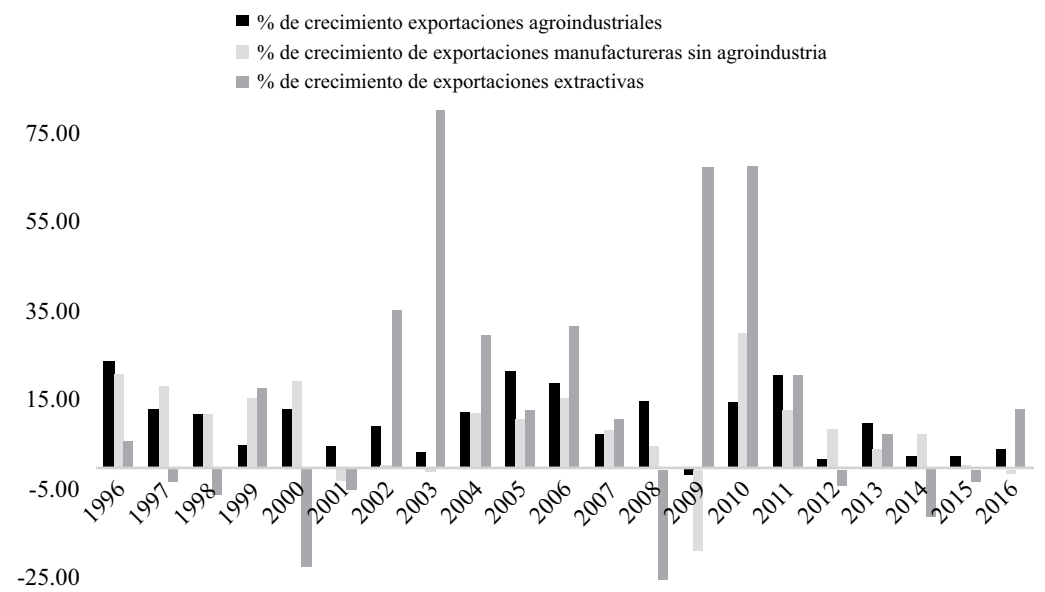

Fuente: Elaboración propia con datos de INEGI. 
Por otra parte, en lo que se refiere al empleo subordinado y remunerado, la Gráfica 5 describe su evolución de 1995 a 2016. Se considera este tipo de empleo, ya que es el que genera en gran medida las actividades de exportación. Como se ve, presenta una tendencia positiva en todo el periodo, con una tasa de crecimiento regular promedio de $2.92 \%$, un poco más de la tasa promedio de crecimiento del PIB. Si tomamos en cuenta la dinámica de crecimiento de las exportaciones analizadas en relación al comportamiento del empleo, podemos darnos cuenta que mientras el valor de las exportaciones presenta un ritmo de crecimiento alto, parece no tener el impacto deseado en la generación de empleos.

\section{Gráfica 5}

Número de empleados subordinados y remunerados, 1995-2016

(Millones de personas)

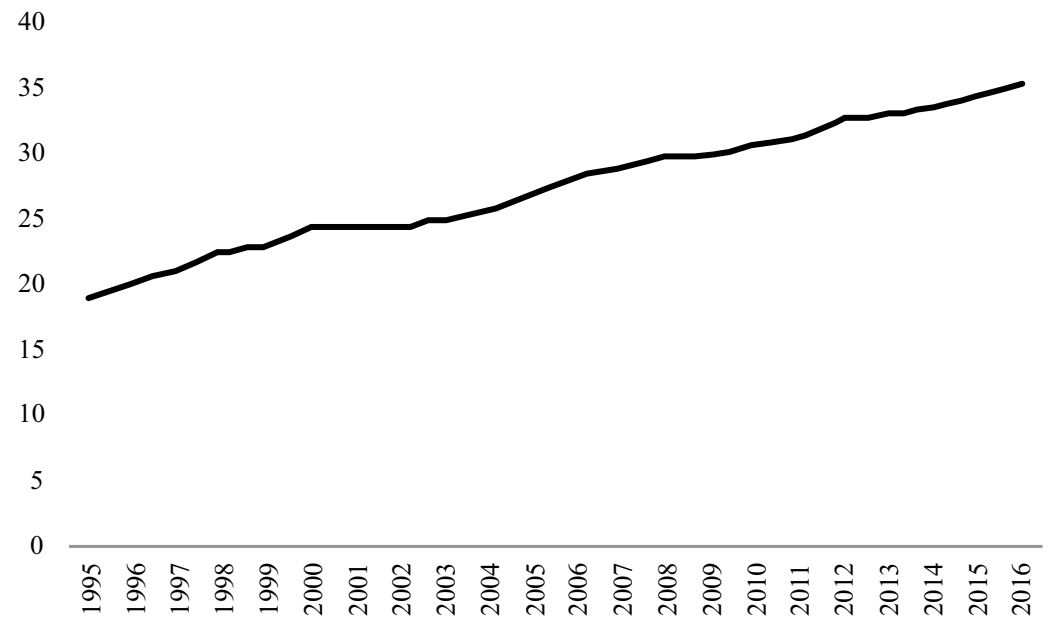

Fuente: Elaboración propia con base en datos de INEGI.

El punto de interés radica entonces en determinar en qué medida las exportaciones analizadas han contribuido en la generación de empleos, si han tenido un peso decisivo o, por el contrario, no son fuentes importantes de empleo, ya que presentan un uso intensivo de capital y bajo en mano de obra, lo que representaría en última instancia un factor negativo para lograr que el derrame de dicho sector se extienda al resto de la economía.

\section{El modelo de regresión lineal múltiple}

Dentro del modelo económico de apertura que se ha venido implementando en el país a raíz del cambio estructural experimentado en los años ochenta, uno de los argumentos que frecuentemente se ha utilizado para defender el cambio es el de la creación de empleos bien 
remunerados. En este trabajo nos limitamos a la comprobación de esta hipótesis solo en el tema de la generación de empleos remunerados, de tal manera que la pregunta a si se generan o no empleos bien remunerados queda abierta.

Para cumplir con el objetivo se establece como variable dependiente el empleo remunerado en número de personas. Como variables independientes se tomaron en cuenta las exportaciones agroindustriales, las manufactureras (con maquila y sin agroindustria) y las extractivas.

La información que proporciona INEGI acerca de las exportaciones manufactureras incluye a las exportaciones agroindustriales. La literatura empírica señala que la industria de la manufactura se encuentra desligada del resto de la economía y que ha perdido dinamismo, generando en consecuencia, un menor ritmo de nuevos empleos. La separación de las exportaciones de manufacturas en agroindustriales y manufacturas con maquila y sin agroindustria, se debe a que queremos enfatizar que las exportaciones agroindustriales han mostrado un dinamismo marcado, que se ha visto reflejado en una mayor generación de empleos y valor agregado, además de un mayor encadenamiento hacia el mercado interno, ya que su principal insumo proviene del sector agropecuario. Esto representa un enorme campo de oportunidades en las exportaciones agroindustriales, a pesar de que su valor de exportación es mucho menor al del resto de las actividades manufactureras. Se constata que la generación de valor agregado en actividades extractivas, así como agropecuarias, tienen tres grandes ventajas: el incremento en el valor de las exportaciones, mayor generación de empleo y estímulo al mercado interno a través del incentivo que sobre la inversión tiene la demanda agregada; esta combinación de factores hace altamente atractivo el estímulo para el fortalecimiento de este tipo de actividades en la consecución de objetivos primordiales en materia económica.

\subsection{Metodología}

A un nivel de confianza de 95 por ciento, mediante la prueba de significancia individual para un modelo de MCO, el modelo queda expresado como: ${ }^{1}$

$$
E_{t-1}=B_{0}+B_{1} X^{X a i_{t-1}}+B_{2} X m_{t-1}+B_{3} \text { Xextractivas }_{t-1}+e
$$

donde $E_{t-1}$ es el número total de hombres y mujeres ocupados que perciben una remuneración por su trabajo en el periodo $t-1 ; X a i_{t-1}$ representa el monto de exportaciones agroindus-

\footnotetext{
Cabe destacar que en un primer momento se incluyeron las exportaciones petroleras y agropecuarias, sin embargo, resultaron ser no significativas, por lo que se omitieron del modelo. Además, se ensayaron varios modelos alternativos. Para cumplir con los supuestos clásicos se realizó un rezago a todas las variables, dicho rezago, al realizarse en todas las variables, tanto dependiente como independientes, no representa ningún cambio teórico-económico en su formulación, por lo que resulta en una regresión equivalente ( $\sin$ rezagos) comprendida de 1995 a 2015.
} 
triales en millones de dólares en dicho periodo. $X m_{t-1}$ son las exportaciones manufactureras con maquila y sin la agroindustria en millones de dólares en t-1; Xextractivas ${ }_{\mathrm{t}-1}$ contiene las exportaciones extractivas en millones de dólares en $t-1 . B_{0}$ es la constante de la regresión, la cual representa el número de ocupados remunerados que no tienen relación con las exportaciones analizadas; $B_{1}, B_{2}$ y $B_{3}$, son los parámetros respectivos de cada variable independiente; y finalmente $e$ recoge los residuos del modelo.

Lo que el modelo plantea es que las variaciones en los montos de exportación deben explicar los cambios en el empleo del país. Si lo que se afirma en el discurso es verdadero, una mayor actividad exportadora deberá verse reflejado en un incremento en el empleo. Cabe destacar que se toma el empleo total para observar el grado de encadenamiento que existe entre el sector exportador y la economía interna. Según autores como Bulmer (1998), América Latina, incluyendo a México, ha tenido históricamente un sector exportador de enclave, es decir, que no se encuentra vinculado al mercado interno, de tal manera que la dinámica del sector no se ve derramada en el resto de la economía.

El modelo toma datos de exportación proporcionados por el Sistema de Cuentas Nacionales (SCN) y datos del empleo derivados de la Encuesta Nacional de Ocupación y Empleo (ENOE) de INEGI. Las exportaciones se encuentran expresadas en millones de dólares FOB, ${ }^{2}$ que es la unidad de medida que se utiliza con frecuencia en análisis del sector exportador por la naturaleza in situ de la actividad; mientras que el empleo se presenta en número de personas remuneradas.

Con el fin de comprobar las propiedades de las series de datos recabadas, se realizaron las pruebas de normalidad, así como de heteroscedasticidad, multicolinealidad, autocorrelación para verificar que el modelo cuenta con la forma funcional correcta (Véase el Anexo).

La alta asociación entre las variables explicativas se debe a la naturaleza económica de las exportaciones, ${ }^{3}$ esta se puede verificar en la matriz de correlación que se presenta en el cuadro 2.

\section{Cuadro 2}

Matriz de correlación

\begin{tabular}{lllll}
\hline & Empleo $_{t-1}$ & \multicolumn{1}{c}{ Xai $_{t-1}$} & Xextractivas $_{t-1}$ & \multicolumn{1}{c}{ Xm $_{t-1}$} \\
\hline Empleo $_{\mathrm{t}-1}$ & 1 & 0.971818 & 0.881642 & 0.979666 \\
Xai $_{\mathrm{t}-1}$ & 0.971818 & 1 & 0.95844 & 0.986707 \\
Xextractivas $_{\mathrm{t}-1}$ & 0.881642 & 0.95844 & 1 & 0.94352 \\
Xm $_{\mathrm{t}-1}$ & 0.979666 & 0.986707 & 0.94352 & 1 \\
\hline
\end{tabular}

Fuente: Elaboración propia.

2 Free On Board. Es un Incoterm, donde el vendedor corre con los gastos de transporte desde la unidad productiva hasta el buque de embarque acordado con el comprador.

3 Como se señala en Gujarati (2010), en los fenómenos económicos frecuentemente se observan asociaciones fuertes entre las variables explicativas debidas a la imposibilidad de la separación de la relación práctica existente entre ellas. Dado que las variables explicativas son significativas, el problema de la multicolinealidad puede ser aceptado, sin que por ello el modelo deje de ser MELI. 


\subsection{Resultados e interpretaciones}

Los resultados de la regresión se presentan en el Cuadro 3.

\begin{tabular}{|c|c|}
\hline \multicolumn{2}{|c|}{$\begin{array}{c}\text { Cuadro } 3 \\
\text { Resultados de la regresión }\end{array}$} \\
\hline $\begin{array}{l}\mathrm{R}^{2} 0.989216 \\
\mathrm{R}^{2} \text { ajustada } 0.987312\end{array}$ & \\
\hline Parámetros: & \\
\hline $\begin{array}{l}\mathrm{B}_{0} \\
\mathrm{~B}_{1} \\
\mathrm{~B}_{2} \\
\mathrm{~B}_{3} \\
\end{array}$ & $\begin{array}{c}15910149 \\
972.5947 \\
39.93 \\
-1580.636 \\
\end{array}$ \\
\hline
\end{tabular}

Fuente: Elaboración propia con base en los resultados de la regresión.

La bondad de ajuste del modelo es del 98.73\%, el modelo alcanza a explicar casi en su totalidad los movimientos registrados en el empleo con la constante y las variables independientes.

La constante nos dice que, si las exportaciones agroindustriales, extractivas y manufactureras fueran cero, se tendrían en el país 15.91 millones de empleos. Es decir, son empleos que se encuentran (en su mayoría) en el mercado interno y que no tienen relación directa con las exportaciones. ${ }^{4}$ En el periodo de estudio, tenemos que, en promedio, 27.1 millones de personas estuvieron ocupadas y remuneradas.

El parámetro $\mathrm{B}_{1}$ indica que por cada incremento en un millón de dólares en exportaciones agroindustriales se crearon en promedio 973 empleos remunerados. $B_{2}$ nos indica que, por cada incremento en un millón de dólares en las exportaciones manufactureras, se crearon en promedio 40 empleos. Finalmente, $\mathrm{B}_{3}$ revela una relación inversa entre el comportamiento de las exportaciones extractivas y el empleo, en promedio, por cada millón de dólares en que se incrementaron las exportaciones de este sector, el número de empleos remunerados retrocedió en 1580. En el análisis conjunto de los resultados se destaca que el 58.7\% de los empleos remunerados no tienen relación alguna con las exportaciones señaladas.

Cabe mencionar que no se tomaron en cuenta las exportaciones petroleras y las agropecuarias debido a que no fueron significativas en el modelo, es decir, no tienen un gran

4 Como se mencionó, en el proceso de modelación, se ensayaron varios modelos alternativos, entre los cuales se tomaron en consideración las exportaciones petroleras y las agropecuarias, las cuales no explican los cambios registrados en el empleo principalmente por la rigidez laboral en el sector petrolero, y por la baja utilización de mano de obra remunerada en el sector agropecuario, de tal forma que la constante engloba -en mayor medida- el empleo que se encuentra en el mercado interno y de manera accesoria a las actividades vinculadas a la importación. 
impacto en los cambios en el empleo remunerado. De las exportaciones consideradas, vemos que son las exportaciones agroindustriales las que generan un mayor número de empleos remunerados, muy por encima de las exportaciones manufactureras. La literatura empírica al respecto respalda lo aquí dicho, ya que, debido a las características de la manufactura, el cual es intensivo en capital y no en mano de obra, no genera empleos como si lo hace la industria agroindustrial, la cual, en ese aspecto, es menos intensiva en capital y más intensiva en mano de obra que las manufacturas. Un resultado interesante es el de la industria de exportación extractiva, el signo negativo del coeficiente revela que no siempre un incremento en el monto monetario de las exportaciones va aparejado de un mayor volumen de exportación y empleo. En otras palabras, el signo negativo revela una combinación de incrementos en el precio de las exportaciones extractivas y una baja promedio en el volumen exportado. ${ }^{5}$ De esta manera, aunado a que la industria extractiva de exportación crea un bajísimo grado de empleos en el país, ${ }^{6}$ las condiciones no han permitido un crecimiento del empleo positivo. Es necesario que la industria extractiva de exportación cree valor agregado en el país, lo cual permitiría un impacto positivo en el empleo y mayor encadenamiento con el mercado interno. ${ }^{7}$ Lo anterior se refuerza en el ámbito de las exportaciones agropecuarias, si tomamos en cuenta por un lado que no fueron significativas en la generación de empleo y por otro lado consideramos la alta significancia de las exportaciones agroindustriales en el empleo, esto muestra claramente como la creación de valor agregado en bienes primarios genera no solamente un mayor valor de exportación, sino a su vez también tiene un impacto positivo en la generación de empleos.

\section{Conclusiones}

El modelo de crecimiento hacia afuera que se ha impulsado desde la década de los ochenta ha quedado a deber en los resultados obtenidos, no ha generado los empleos suficientes como en la teoría se prevé. Más de la mitad de los empleos en el país no tienen ninguna relación con el sector de exportación. Como se ha podido probar mediante el modelo de regresión, la manufactura ya no genera las mismas cantidades de fuentes de empleo que antes, a pesar

5 Según datos de Investing.com, el precio de la plata (uno de los subsectores más importantes de la industria extractiva), de 2003 a 2017, presentó una tendencia positiva, con un promedio de crecimiento anual en el precio de $3.24 \%$.

6 Los datos que presenta la ENOE son reveladores al respecto, en promedio de 2000 a 2016 el empleo que generó la industria extractiva promedió $1.24 \%$ del total de empleos remunerados.

7 Bajo las condiciones actuales del sector extractivo, existen pocos incentivos para generar mayor valor agregado en sus productos, ya que el precio internacional ha evolucionado favorablemente para continuar con la misma dinámica. 
de ser el componente principal de nuestras exportaciones, el capital intensivo que se utiliza en el sector, si bien la ha vuelto altamente productiva, no ha permitido la estimulación del empleo. Además, como se sostiene en la literatura, los productos exportados tienen un alto contenido de insumos importados, lo que presenta dos desventajas: dependencia hacia el exterior y bajo encadenamiento productivo hacia el mercado interno. Se observa, en cambio, un enorme campo de oportunidades en las exportaciones agroindustriales, al separarlas de la manufactura, se encuentra que son grandes generadoras de empleo, a pesar de que su valor de exportación es mucho menor al del resto de las actividades manufactureras, su fomento traería la enorme ventaja de impulsar a la vez el sector exportador y fortalecer, vía empleo, la demanda agregada en el mercado interno.

La industria extractiva presenta la característica de crear un bajísimo número de empleos, su valor de exportación ha crecido de manera exponencial por la tendencia positiva del precio de los bienes que la componen y no por el volumen de exportación, el cual obedece a las características de la demanda internacional. Esto ha ocasionado una relación inversa entre el comportamiento del valor de las exportaciones extractivas respecto al comportamiento del empleo.

\section{Referencias bibliográficas}

Baltazar, J. y Ramos, M. (2014), "México: Comercio Exterior, Producto y condición de MarshallLerner”, Tiempo Económico, vol. IX, núm. 27, pp. 5-26.

Bulmer, V. (2003), La Historia Económica de América Latina Desde la Independencia, Fondo de Cultura Económica, México.

Bustamante, J. y Sánchez, B. (2017). "Perspectiva empresarial en el contexto del modelo de desarrollo exportador mexicano 1994-2014”, Análisis Económico, vol. XXXII, núm. 79, pp. 99-124.

Calderón, C. y Sánchez, I. (2012), “Crecimiento económico y política industrial en México”, Problemas del Desarrollo, vol. 43, núm. 170, pp. 125-154.

— G García, R. y Cruz, S. (2017). "Sincronización comercial e industrial en el TLCAN, un estudio de sincronización de fase”, Economía. Teoría y Práctica, núm. 46, pp. 5-40. DOI: http://dx.doi. org/10.24275/ETYPUAM/NE/462017/Calderon

_ Ochoa, G. y Huesca, L. (2017), "Mercado laboral y cambio tecnológico en el sector manufacturero mexicano (2005-2014)", Economía, Sociedad y Territorio, vol. XVII, núm. 54, pp. 523-560. DOI: http://dx.doi.org/10.22136/est002017958

Carbajal, Y. y Almonte, L. (2017), "Empleo manufacturero en la Región Centro de México. Una estimación por gran división”, Contaduría y Administración, vol. 62, núm. 3, pp. 880-901. DOI: http://dx.doi.org/10.1016/j.cya.2017.04.004

Cermeño, R. y Rivera, H. (2016), "La demanda de importaciones y exportaciones de México en la era del TLCAN: Un enfoque de cointegración”, El Trimestre Económico, vol. LXXXIII(1), núm. 329 , pp. 127-147.

Cisneros, C. y Guerrero, M. (2014), “El sector manufacturero en México (1994-2012): Una restricción externa al crecimiento", Tiempo Económico, vol. XI, núm. 28, pp. 22-39. 
Delgado, R. y Márquez, H. (2007). “Para entender la migración a Estados Unidos: El papel de la fuerza de trabajo barata mexicana en el mercado laboral transnacional" Problemas del Desarrollo, vol. 38, núm. 149, pp. 11-34.

Fujii, G. y Cervantes, M, (2010). "Liberalización comercial y empleo en México", Revista de Economía Mundial, núm. 26, pp. 107-133. Disponible en: http://www.redalyc.org/articulo.oa?id=86618030005

Gómez, C. (2012), "Las exportaciones de México a los Estados Unidos. Su importancia y principales determinantes, 1993-2011", Tiempo Económico, vol. VII, núm. 20, pp. 71-80.

_ (2013), “¿Ha contribuido el TLCAN a la diversificación de las exportaciones mexicanas?”, Tiempo Económico, vol. VIII, núm. 25, pp. 57-70.

— y González, J. (2017), "Competencia y competitividad de las exportaciones de México y China en el mercado estadounidense: nueva evidencia", México y la Cuenca del Pacífico, vol. 6, núm. 16, pp. 79-105.

Guillén, H. (2013), "México: de la sustitución de importaciones al nuevo modelo económico", Comercio Exterior, vol. 63, núm. 4, pp. 34-60.

Gujarati, D. y Porter, D. (2009), Econometría (Quinta ed.). México: McGraw-Hill.

64 Juárez, C. y Ángeles, G. (2011), "Determinantes de la inversión extranjera directa en la República Mexicana (1994 - 2007)”, Tiempo Económico, vol. VI, núm. 19, pp. 25-40.

Lechuga, J. y Varela, M. (2001), “Empleo manufacturero en México, 1990-1998”, Análisis Económico, vol. XVI, núm. 33, pp. 215-234.

Loría, E. y Brito, L, (2005). "El impacto de la inversión extranjera directa en el empleo sectorial en México: un análisis prospectivo", Análisis Económico, vol. XX, núm. 44, pp. 5-35.

Montero. R (2011), Efectos fijos o aleatorios: test de especificación. Documentos deTrabajo en Economía Aplicada. Universidad de Granada. España Disponible en http://www.ugr.es/ montero/ matematicas/especificacion.pdf

Rangel, C. (2015), "México en el TLCAN, 20 años después y el futuro de la política comercial", Tiempo Económico, vol. X, núm. 31, pp. 7-24.

Ríos, E; Contreras, I. y Bustos, R. (2017), "Local and Global productivity and their effects on value added in Mexico, (1993-2015): The case of industrial manufacturing sub branches", ECORFAN Journal, vol.3, núm. 5, pp. 76-89.

Vargas, M. (2003), “Industria maquiladora de exportación. ¿Hacia donde va el empleo?”, Papeles de Población, vol. 9, núm. 37, pp. 243-259.

Vázquez, J. y Avendaño, B. (2012). ¿Modelo de crecimiento exportador o modelo de estancamiento exportador? El caso de México, 1961-2010, Investigación Económica, vol. LXXI, núm. 282, Octubre-Diciembre, pp. 93-108.

Villareal, R. y Ramos, R. (2001). La apertura de México y la paradoja de la competitividad: hacia un modelo de competitividad sistémica, Comercio Exterior, Septiembre, pp. 772-788. 
Anexo

Pruebas de estabilidad y forma funcional

\begin{tabular}{cc}
\hline Prueba & Resultados \\
\hline Jarque Bera & 0.0501 \\
ARCH & Prob. F 0.5815; \\
Prob. Chi-Square 0.5575 \\
Regresión alternativa) & 0.98536 \\
Durbin-Watson & 1.89 \\
\hline
\end{tabular}

Fuente: Elaboración propia. 